\title{
ITERATIVE DIPOLE MOMENT METHOD FOR THE INTERACTION OF MULTIPLE DIELECTROPHORETIC PARTICLES IN AN AC ELECTRICAL FIELD *
}

\author{
Chuanchuan Xie ${ }^{(1)}$, Bo Chen*(1) ${ }^{(1)}$ Liu $^{(1)}$, Han Chen ${ }^{(1)}$, Jiankang Wu ${ }^{(2)}$ \\ ${ }^{1}$ (School of Civil Engineering and Mechanics, Huazhong University of Science and Technology, Wuhan, China, 430074) \\ ${ }^{2}$ (Wenhua College, Wuhan, China, 430074)
}

\begin{abstract}
Dielectrophoresis (DEP) for bio-particle manipulation has been drawing much attention in recent years. The equivalent dipole moment method (EDM) has widely been used to calculate DEP forces on single particle, but this method falls short to describe the interaction between neighboring particles. The Maxwell stress tensor method (MST) is theoretically rigorous for particle interaction, but its complicated numerical computation makes it difficult to implement in practice. In this paper, an iterative dipole moment method (IDM) is presented to investigate the interaction of multiple dielectrophoretic particles in a two-dimensional AC electric field. Without cumbersome numerical computation, the inter-particle forces, the particle trajectories and chain patterns calculated by the IDM method are found to be well consistent with those by the MST method for some published results and experimental observations. Furthermore, it is found that the final stable particle chain patterns strongly depend on the initial configuration of the particle distribution. An arbitrary small disturbance to the particle locations may lead to dramatically different motion trajectories and final particle chains. By tuning the frequency of AC field, the particles can be transformed between positive DEP particles and negative DEP particles, which results in different particle chains as well.
\end{abstract}

Key words: Dielectrophoresis (DEP); Equivalent dipole moment (EDM); Particle interaction;

Maxwell stress tensor (MST); Iterative dipole moment (IDM)

\section{INTRODUCTION}

Dielectrophoresis (DEP) is one of the most popular techniques for bio-particle manipulation in microfluidic systems. The interactive DEP forces can lead to particle chains observed in particle assembly experiments [1]. The particle chain phenomenon is the basis of DEP assembly technique, one of the most important techniques to assemble micro/nano materials in microfluidic applications. For instance, DEP can be used to assemble hematopoietic stem cells into a multilayered structure for studying the process of blood cell production [2], and fabricate biosensors using DNA/protein molecules [3].

The dynamics of DEP has been studied using the equivalent dipole moment method (EDM) [4]. The EDM method provides good estimations of DEP forces only in the dilute limit of the particle density. However, it loses validity and can produce erroneous results in some cases where particles create significant spatial non-uniformities in the electric field. For instance, the particles are near an electrode, two or more particles are close to each other or clustered together. The DEP force is calculated based on the electric field without the effect of other particles in the EDM approximation. The Maxwell stress tensor

Supported by National Science Foundation of China, No. 11572139

Corresponding author: Dr. Chen Bo, email: chbo76@hust.edu.cn 
(MST) method has been considered as the most rigorous method to determine the DEP forces in general [5-7]. The interactions of finite-size particles by the MST method under DC electric field [8-17] or AC electric field [18-20] have been well studied. The MST method is theoretically accurate for particle interaction in an electric field, but it requires complicated numerical computations for solving differential equations of electrical fields. The numerical errors are difficult to control and meanwhile the cost in computation is huge in cases of multiple particles. It is not feasible to study the interactions of multiple particles using the MST method. When the electric field induced by the polarized particle is comparable to the applied electric field, the induced electric field together with applied electric field may cause non-homogenous polarization of the particle. In such a case, the multipolar approximation [21-24] is used to recreate the electric potential around the particle. Using the multipolar approximation method, Nakajima and Hernandez $[25,26]$ studied the electric field distribution around the particles of the particle chains.

In this paper, we will present an iterative dipole moment method (IDM) to calculate the interactive forces of multiple DEP particles in AC field, as well as motions. The accuracy, convergence and simplicity of IDM method have been demonstrated in the DC field [27-29]. The local electrical field is repeatedly corrected in this method to obtain accurate interactive forces in comparison with MST method, as validated in the following sections. The interactive forces on particles calculated by the proposed IDM method agree very well with those by the MST method, the particle trajectories and the final particle chains are in good agreement with the published results and consistent with experimental results [30,31]. We investigate the patterns of particle chains and the particle trajectories according to different initial particle positions and frequency of electric field. The sensitivity of the final particle chains to the initial particle locations is revealed by applying a minimal disturbance in the initial locations. By tuning the frequency of the AC electric field, totally different results are obtained in the multiple particles interaction.

\section{THEORY}

2.1. IDM method for calculating particle interactions in two-dimensional AC electric field

Consider $\mathrm{N}$ neutral particles randomly suspended in an incompressible Newtonian fluid in a region of square $(L \times L)$. The particles are located at $\left(\mathrm{x}_{i}, \mathrm{y}_{i}\right)(i=1,2, \cdots, \mathrm{N})$, respectively. An AC electric field $\tilde{\boldsymbol{E}}_{0}$ is applied, and the polarized charge on the $i$ th particle is approximated as a two-dimensional point-dipole moment written as follows [32]:

$$
\tilde{\boldsymbol{p}}_{i}=2 \pi a_{i}^{2} \varepsilon_{f} K_{i}(\omega) \tilde{\boldsymbol{E}}_{0}
$$

where $\tilde{\boldsymbol{p}}_{i}$ denotes the complex dipole moment, $a_{i}$ is the radius of the $i$ th particle, $K_{i}(\omega)$ is the Clausius-Mossotti factor, given by

$$
K_{i}(\omega)=\frac{\tilde{\varepsilon}_{p i}-\tilde{\varepsilon}_{m}}{\tilde{\varepsilon}_{p i}+\tilde{\varepsilon}_{m}}
$$

where $\tilde{\varepsilon}_{p i}=\varepsilon_{p i}-j \sigma_{p i} / \omega$ and $\tilde{\varepsilon}_{f}=\varepsilon_{f}-j \sigma_{f} / \omega$ is the complex permittivities of the $i$ th particle and fluid, respectively, $j=\sqrt{-1}$, and the superscript " $\sim$ " denotes the complex variables. $\varepsilon_{p i}$ and $\sigma_{p i}$ are the permittivity and conductivity of the $i$ th particle, respectively, $\varepsilon_{f}$ and $\sigma_{f}$ are the permittivity and 
conductivity of the fluid, respectively. $\omega=2 \pi f$ is the angular frequency of the AC electric field. The dipole moment will induce a local electric field, called dipole-induced field in this work, written as follows

$$
\tilde{\varphi}=\frac{\tilde{\boldsymbol{p}} \cdot \boldsymbol{r}}{2 \pi \varepsilon_{f} r^{2}}
$$

where $\boldsymbol{r}$ is the position vector originated from the ith particle location $\left(x_{i}, y_{i}\right), r=|\boldsymbol{r}|$. When the particles are close to each other, the local electric field around the $j$ th particle is corrected by an additional electrical field from the $i$ th particle written as follows

$$
\begin{gathered}
\tilde{\varphi}_{i j}=\frac{\tilde{\boldsymbol{p}}_{i} \cdot \boldsymbol{r}_{i j}}{2 \pi \varepsilon_{f} r_{i j}^{2}}=\frac{a_{i}^{2} K_{i}(\omega) \cdot\left(\tilde{E}_{0 x}\left(x_{j}-x_{i}\right)+\tilde{E}_{0 y}\left(y_{j}-y_{i}\right)\right)}{r_{i j}^{2}} \\
\left(\tilde{E}_{x}\right)_{i j}=-\frac{\partial \varphi_{i j}}{\partial x}=-a_{i}^{2} K_{i}(\omega)\left[\frac{\tilde{E}_{0 x}}{r_{i j}^{2}}-\frac{2\left(\tilde{E}_{0 x}\left(x_{j}-x_{i}\right)+\tilde{E}_{0 y}\left(y_{j}-y_{i}\right)\right)\left(x_{j}-x_{i}\right)}{r_{i j}^{4}}\right] \\
\left(\tilde{E}_{y}\right)_{i j}=-\frac{\partial \varphi_{i j}}{\partial y}=-a_{i}^{2} K_{i}(\omega)\left[\frac{\tilde{E}_{0 y}}{r_{i j}^{2}}-\frac{2\left(\tilde{E}_{0 x}\left(x_{j}-x_{i}\right)+\tilde{E}_{0 y}\left(y_{j}-y_{i}\right)\right)\left(y_{j}-y_{i}\right)}{r_{i j}^{4}}\right]
\end{gathered}
$$

where $r_{i j}=\left|\boldsymbol{r}_{i j}\right|=\sqrt{\left(x_{j}-x_{i}\right)^{2}+\left(y_{j}-y_{i}\right)^{2}}$, the vector $\boldsymbol{r}_{i j}$ is pointed from ith particle to the $j$ th particle, $\tilde{E}_{0 x}$ and $\tilde{E}_{0 y}$ are the x-component and y-component and the electric field, respectively. The first corrected electric field around the $j$ th particle center $\tilde{\boldsymbol{E}}_{j}^{(1)}$ can be expressed as follows:

$$
\tilde{\boldsymbol{E}}_{j}^{(1)}=\tilde{\boldsymbol{E}}_{j}^{(0)}+\sum_{i=1, i \neq j}^{N} \tilde{\boldsymbol{E}}_{i j}^{(0)}
$$

where $\tilde{\boldsymbol{E}}_{j}^{(0)}$ is the applied electric field at the $j$ th particle center, $\tilde{\boldsymbol{E}}_{i j}^{(0)}$ is the additional electrical field resulted from the dipole-induced field of the $i$ th particle, calculated by Eq.(5). The first corrected electric field will induce a new dipole $\tilde{\boldsymbol{E}}_{i}^{(1)}$ and a new dipole-induced electric field $\tilde{\boldsymbol{E}}_{i j}^{(1)}$, and the second corrected electric field $\tilde{\boldsymbol{E}}_{j}^{(2)}$ is obtained in the same way. The local electric field, dipole moment and the dipole-induced electric field will be iteratively corrected, until a converged electric field is obtained, as shown in Fig.(1), where $n$ denotes the iteration number of the electric field correction, and $\tau$ denotes the convergence criteria of iteration error.

$$
\left|\frac{\tilde{E}_{x}^{(n+1)}-\tilde{E}_{x}^{(n)}}{\tilde{E}_{x}^{(n+1)}}\right| \leq \tau, \quad\left|\frac{\tilde{E}_{y}^{(n+1)}-\tilde{E}_{y}^{(n)}}{\tilde{E}_{y}^{(n+1)}}\right| \leq \tau
$$

for all particles.

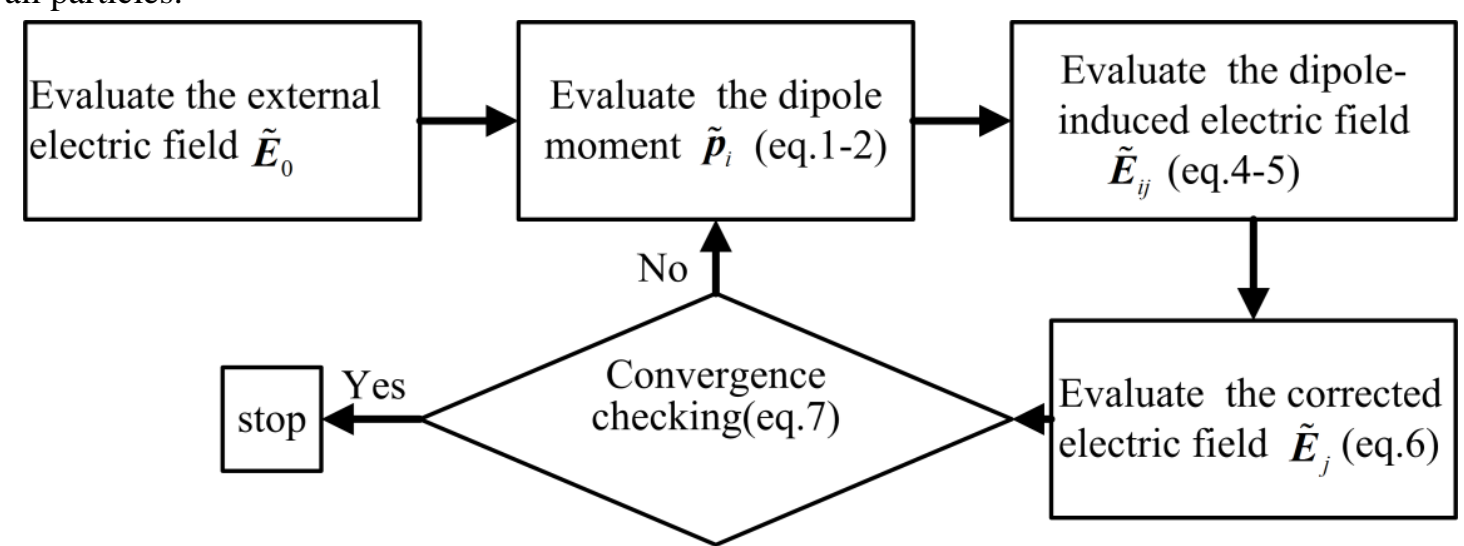

Fig .1 Flow diagram of the IDM method to get the convergent value of electric field. 
When the converged solution of the electric field are obtained, the time-averaged DEP force of particles can be written as [32]

$$
\left\langle\boldsymbol{F}_{\text {dep }}\right\rangle=\pi a^{2} \varepsilon_{f} \operatorname{Re}[K(\omega)] \nabla|\tilde{\boldsymbol{E}}|^{2}
$$

And the time-averaged first order electrorotation torque on the particle is

$$
\left\langle\boldsymbol{\Gamma}_{\text {dep }}\right\rangle=\frac{1}{2} \operatorname{Re}[\boldsymbol{p} \times \tilde{\boldsymbol{E}}]
$$

The motion of the particles is governed by the kinematic equation as follows

$$
m_{p} \frac{d^{2} \boldsymbol{r}_{p}}{d t^{2}}=\boldsymbol{F}_{d e p}+\boldsymbol{F}_{d}
$$

where $m_{p}, \boldsymbol{r}_{p}$ is the mass and the position vector of particles, respectively. $\boldsymbol{F}_{d}$ is the Stokes drag of fluid [30], given by

$$
\boldsymbol{F}_{d}=\frac{16 \pi \eta a_{l}}{2 \ln \left(2 a_{l} / a\right)-1}(\boldsymbol{u}-\boldsymbol{v})
$$

where the fluid viscosity is $\eta=10^{-3} \mathrm{~N} \cdot \mathrm{s} / \mathrm{m}^{2}, a_{l}$ is the length of cylindrical particle perpendicular to the two-dimensional region, and $a_{l}>>a . \boldsymbol{u}, \boldsymbol{v}$ are the velocities of the fluid and particle, respectively. The fluid velocity is assumed to be zero, so equation (12) can be reduced to

$$
\boldsymbol{F}_{d}=8 \pi \eta \boldsymbol{v}
$$

2.2. MST method for calculating interactive forces of particles in AC electric field.

The electric potential is governed by the Gauss's law, given by

$$
\begin{array}{ll}
\nabla \cdot\left(\tilde{\varepsilon}_{f} \nabla \tilde{\varphi}_{f}\right)=0 & \text { in fluid } \\
\nabla \cdot\left(\tilde{\varepsilon}_{p} \nabla \tilde{\varphi}_{p}\right)=0 & \text { in particle }
\end{array}
$$

where $\tilde{\varphi}_{f}$ and $\tilde{\varphi}_{p}$ is the complex potential in the fluid and the particle, respectively. Due to the difference in permittivity, the electric field is discontinuous across the particle-fluid interface where the electric flux condition is applied as $\tilde{\varepsilon}_{p} \cdot \partial \tilde{\varphi}_{p} / \partial n=\tilde{\varepsilon}_{f} \cdot \partial \tilde{\varphi}_{f} / \partial n$. When electrical field is solved $\tilde{E}=-\nabla \tilde{\varphi}$, the time-averaged Maxwell stress tensors on both sides of particle surface are calculated as follows [7]:

$$
\boldsymbol{T}_{p}=\frac{\varepsilon_{p}}{4}\left[\left(\tilde{\boldsymbol{E}}_{p} \tilde{\boldsymbol{E}}_{p}^{\prime}+\tilde{\boldsymbol{E}}_{p}^{\prime} \tilde{\boldsymbol{E}}_{p}\right)-\left|\tilde{\boldsymbol{E}}_{p}\right|^{2} \boldsymbol{I}\right]
$$

for the particle side of the surface, and

$$
\boldsymbol{T}_{f}=\frac{\varepsilon_{f}}{4}\left[\left(\tilde{\boldsymbol{E}}_{f} \tilde{\boldsymbol{E}}_{f}^{\prime}+\tilde{\boldsymbol{E}}_{f}^{\prime} \tilde{\boldsymbol{E}}_{f}\right)-\left|\tilde{\boldsymbol{E}}_{f}\right|^{2} \boldsymbol{I}\right]
$$

for the fluid side of the surface, where $\boldsymbol{I}$ denotes the second-order unit tensor. $\tilde{\boldsymbol{E}}_{f}$ and $\tilde{\boldsymbol{E}}_{p}$ are the complex electric field on the fluid side and the particle side of the particle surface, respectively. ( )' denotes conjugate of a complex number. The time-averaged DEP force of particles is obtained by an integral of MST over the particle surface [17]:

$$
\boldsymbol{F}_{d e p}=\oint\left(\boldsymbol{T}_{f}-\boldsymbol{T}_{p}\right) \cdot \boldsymbol{n} d s
$$


where $\boldsymbol{n}$ is the unit normal vector on the particle surface pointing to the fluid, the time-averaged DEP torque can be written as

$$
\boldsymbol{\Gamma}_{d e p}=\oint a \boldsymbol{n} \times\left[\left(\boldsymbol{T}_{f}-\boldsymbol{T}_{p}\right) \cdot \boldsymbol{n}\right] d s
$$

2.3. The dimensionless governing equations

All the equations above are normalized by the characteristic length $a=5 \mu \mathrm{m}$, potential $\varphi_{0}=10 \mathrm{~V}$, velocity $U_{0}=\varepsilon_{f} \varphi_{0}^{2} /(\eta a)$, time $t_{0}=a / U_{0}$, force $F_{0}=\eta U_{0}$, stress tensor $T_{0}=\eta U_{0} / a$, mass $m_{0}=a \eta / U_{0}[20]$. The normalized equations are expressed as:

$$
\begin{gathered}
\boldsymbol{F}_{d e p i}^{*}=\pi \operatorname{Re}[K(\omega)] \nabla\left|\tilde{\boldsymbol{E}}_{i}^{*}\right|^{2} \\
\Gamma_{d e p i}^{*}=\frac{1}{2} \operatorname{Re}\left[\boldsymbol{p}_{i}^{*} \times \tilde{\boldsymbol{E}}_{i}^{*}\right] \\
\boldsymbol{F}_{d}^{*}=8 \pi \boldsymbol{v}^{*} \\
m_{p}^{*} \frac{d^{2} \boldsymbol{r}_{p}^{*}}{d t^{* 2}}=\boldsymbol{F}_{d e p}^{*}+\boldsymbol{F}_{d}^{*}
\end{gathered}
$$

for the IDM method, and

$$
\begin{array}{cc}
\nabla^{*} \cdot\left(\tilde{\varepsilon}_{f}^{*} \nabla \tilde{\varphi}_{f}^{*}\right)=0 & \text { in fluid } \\
\nabla^{*} \cdot\left(\tilde{\varepsilon}_{p}^{*} \nabla \tilde{\varphi}_{p}^{*}\right)=0 & \text { in particles } \\
\boldsymbol{T}_{p}^{*}=\frac{1}{4}\left[\left(\tilde{\boldsymbol{E}}_{p}^{*} \tilde{\boldsymbol{E}}_{p}^{*}+\tilde{\boldsymbol{E}}_{p}^{\prime *} \tilde{\boldsymbol{E}}_{p}^{*}\right)-\left|\tilde{\boldsymbol{E}}_{p}^{*}\right|^{2} \boldsymbol{I}\right] \\
\boldsymbol{T}_{f}^{*}=\frac{1}{4}\left[\left(\tilde{\boldsymbol{E}}_{f}^{*} \tilde{\boldsymbol{E}}_{f}^{\prime *}+\tilde{\boldsymbol{E}}_{f}^{\prime *} \tilde{\boldsymbol{E}}_{f}^{*}\right)-\left|\tilde{\boldsymbol{E}}_{f}^{*}\right|^{2} \boldsymbol{I}\right] \\
\boldsymbol{F}_{d e p}^{*}=\oint\left(\boldsymbol{T}_{f}^{*}-\boldsymbol{T}_{p}^{*}\right) \cdot \boldsymbol{n} d s^{*} \\
\boldsymbol{\Gamma}_{d e p}^{*}=\oint \boldsymbol{n} \times\left[\left(\boldsymbol{T}_{f}^{*}-\boldsymbol{T}_{p}^{*}\right) \cdot \boldsymbol{n}\right] d s^{*}
\end{array}
$$

for the MST method, where ()$^{*}$ denotes the dimensionless variable, $\tilde{\varepsilon}_{f}^{*}=\tilde{\varepsilon}_{f} / \varepsilon_{f}, \tilde{\varepsilon}_{p}^{*}=\tilde{\varepsilon}_{p} / \varepsilon_{f}$ and $\operatorname{Re}=\rho_{f} a U_{0} / \eta$

\section{Validation of the accuracy of the interactive DEP forces by IDM method}

To verify the accuracy of the IDM method, the DEP force and the electrorotation torque are calculated by the IDM method in a uniform two-dimensional AC electric field are compared with those of the MST method. Two particles $\left(a_{1}=a_{2}=5 \mu \mathrm{m}\right)$ are suspended in a square domain $(L \times L=100 \mu \mathrm{m} \times 100 \mu \mathrm{m})$ filled with fluid, as shown in Fig.2. The voltages are applied on electrodes $\mathrm{AB}$ and $\mathrm{CD}, \tilde{\varphi}_{A B}=\varphi_{0}=10 \mathrm{~V}$ and $\tilde{\varphi}_{C D}=0$. So the dimensionless values $a_{i}^{*}=a_{i} / a=1, L^{*}=L / a=20, \quad \tilde{\varphi}_{A B}^{*}=\tilde{\varphi}_{A B} / \varphi_{0}=1$, and $\tilde{E}_{0}^{*}=\tilde{\varphi}_{A B}^{*} / L^{*}=0.05$. The permittivities of the particle and the electrolyte are $\varepsilon_{p}=2.5 \varepsilon_{0}$ and $\varepsilon_{f}=78 \varepsilon_{0}$, where $\varepsilon_{0}$ is the permittivity in vacuum, the conductivities are $\sigma_{p 1}=5.0 \times 10^{-3} \mathrm{~S} / \mathrm{m}, \sigma_{p 2}=3.0 \times 10^{-4} \mathrm{~S} / \mathrm{m}$, $\sigma_{f}=1.0 \times 10^{-3} \mathrm{~S} / \mathrm{m}$, respectively. $R$ is the half distance between the two particle centers. $\theta$ is the directional angle between the connecting line of the two particle centers and the electrical field (the x-axis). 
The real part of C-M factors of the two particles are shown in Fig.3, where $K_{R}=\operatorname{Re}[K(\omega)]$. The particle is defined as positive particle (or P particle), when $K_{R}>0$, and negative particle (or N particle) when $K_{R}<0$ through this paper. COMSOL Multiphysics is employed to compute the time-averaged DEP forces of the particles by MST and the grids in vicinity of the particle surface have been sufficiently refined to obtain grid-independent numerical solutions. A relative error criterion of $\tau=10^{-3}$ is used in IDM method.

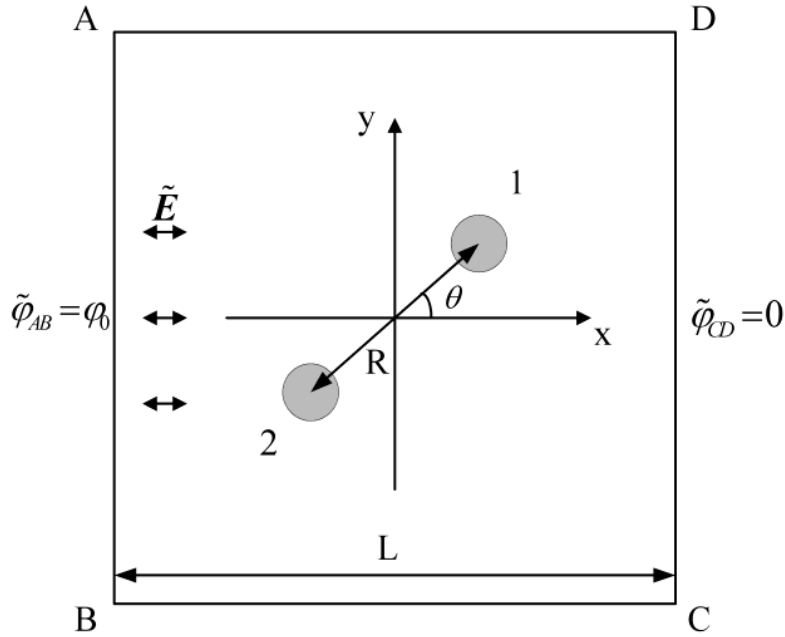

Fig.2 A pair of particles suspended in fluid subjected to an uniform AC electric field parallel to the $\mathrm{x}$ axis. $R$ is the half distance between the two particle centers. $\theta$ is the angle between the connecting line of particle centers and the $\mathrm{x}$-axis.

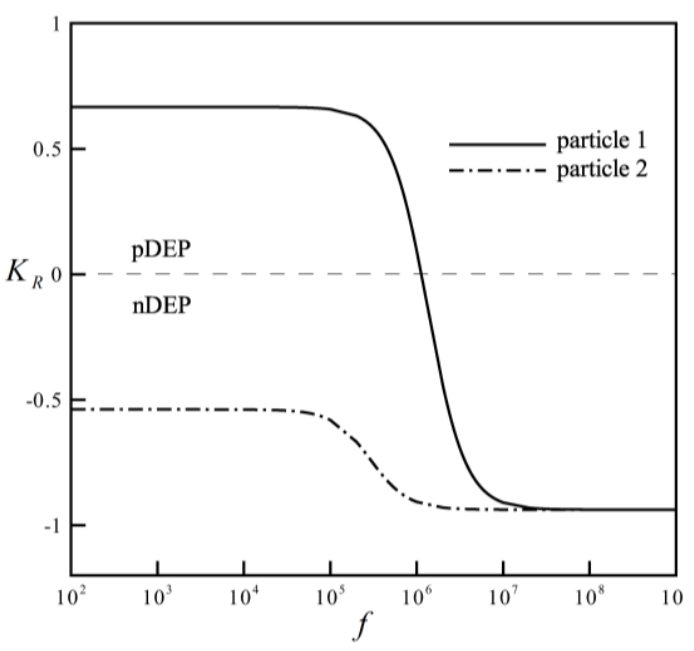

Fig.3 The real part of C-M factors of two particles versus frequency of the $\mathrm{AC}$ electric field. The two particles have the same permittivity $\varepsilon_{p}=2.5 \varepsilon_{0}$ but different conductivities $\sigma_{p 1}=5.0 \times 10^{-3} \mathrm{~S} / \mathrm{m}$, $\sigma_{p 2}=3.0 \times 10^{-4} \mathrm{~S} / \mathrm{m}$

The dependence of DEP forces and electrorotation torque on the distance R is shown in Fig.4 when $\theta=45^{\circ}$ and $f=10^{3} \mathrm{~Hz}$, the particle 1 is positive, the particle 2 is negative, see Fig. 3 . It can be seen that the results of IDM method and MST method are in good agreement as the distance of the two particles varies. Particle interaction is not considered in the IDM method when $n=0$, the IDM method reduces to conventional EDM method, the forces and torques of the particles are zero in a uniform field. It can also be seen that the DEP forces and torques in IDM method are converged very quickly. The results of IDM are much close to those of MST when iteration number $n=2$. The dependence of DEP forces and torque on the frequency of electric field is shown in Fig.5 when $\theta=45^{\circ}$ and $R^{*}=R / a=4$. Again it can be seen that both IDM and MST obtain almost the same DEP forces and torques in different frequencies. A little difference is found in a narrow region of frequency, around $10^{6} \mathrm{~Hz}$ in the present case, where the DEP forces and torques are much close to zero, and rapidly vary. 

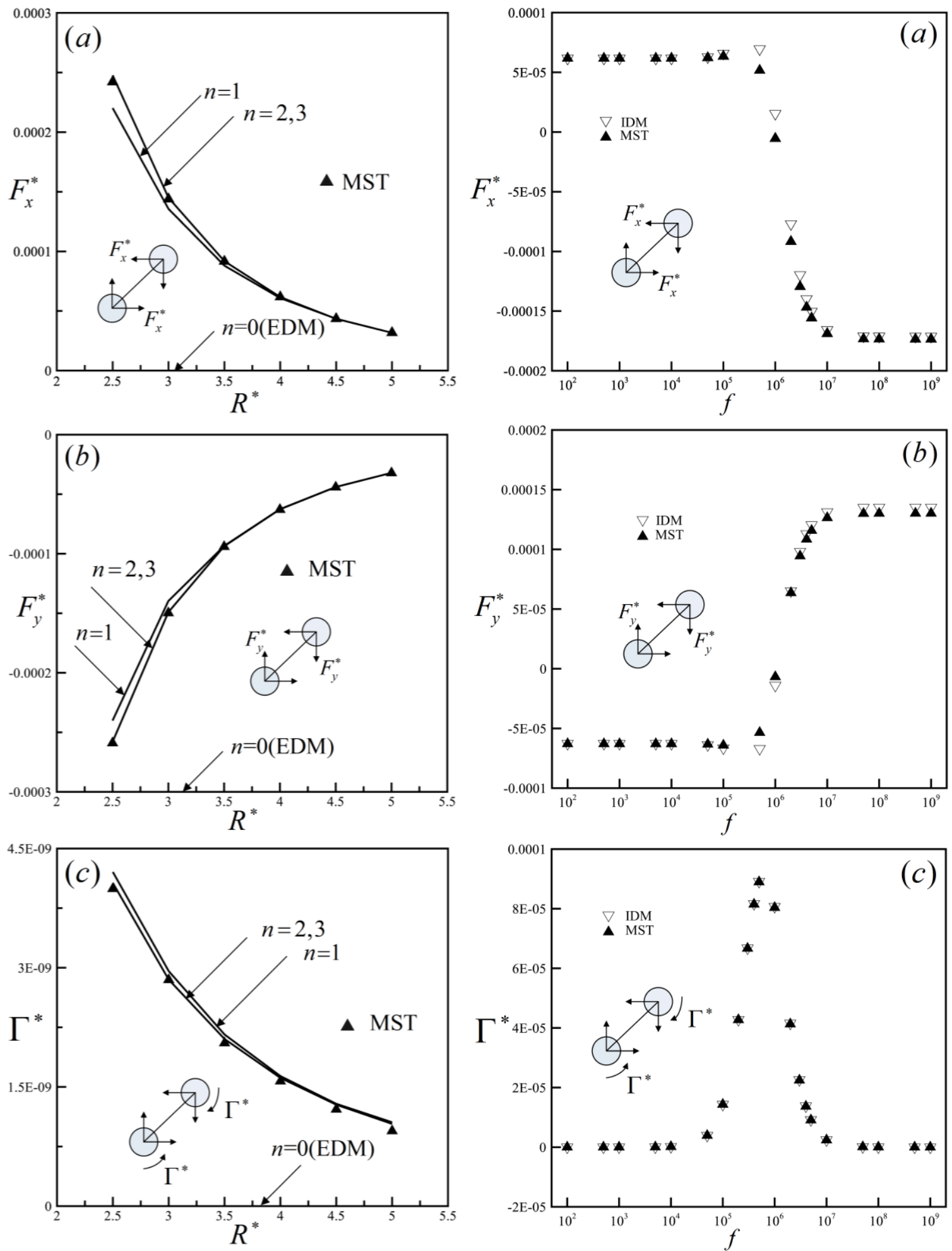

Fig.4 DEP forces and torques versus $R^{*}$, the ratio of distance of two particle centers to particle radius when $\theta=45^{\circ}$ and $f=10^{3} \mathrm{~Hz}$. (a) DEP forces in $\mathrm{x}$ direction, (b) DEP forces in y direction, (c) DEP torques. $n$ is the iteration number of IDM method.

Fig.5 DEP forces and torques versus the frequency of the electrical field when $\theta=45^{\circ}$ and $R^{*}=R / a=4$. (a) DEP forces in x direction, (b) DEP forces in y direction (c) DEP torques. $\nabla$ IDM $\Delta$ MST

The particle trajectories calculated by IDM and MST are compared for different frequencies, when $\theta=45^{\circ}$ 
and $R^{*}=4$, as shown in Fig.6. It can be seen that the particles rotate counterclockwise and finally form a particle chain perpendicular to the electric field at $f=10^{3} \mathrm{~Hz}$, where the particle 1 is $\mathrm{P}$ particle and the particle 2 is $\mathrm{N}$ particle, see Fig.3. The particles rotate clockwise and form a particle chain parallel to the electric field at $f=5 \times 10^{6} \mathrm{~Hz}$, where both two particles are $\mathrm{N}$ particles. As we can see, the particle trajectories by IDM method are consistent with that by MST method, so the feasibility of IDM method is verified.

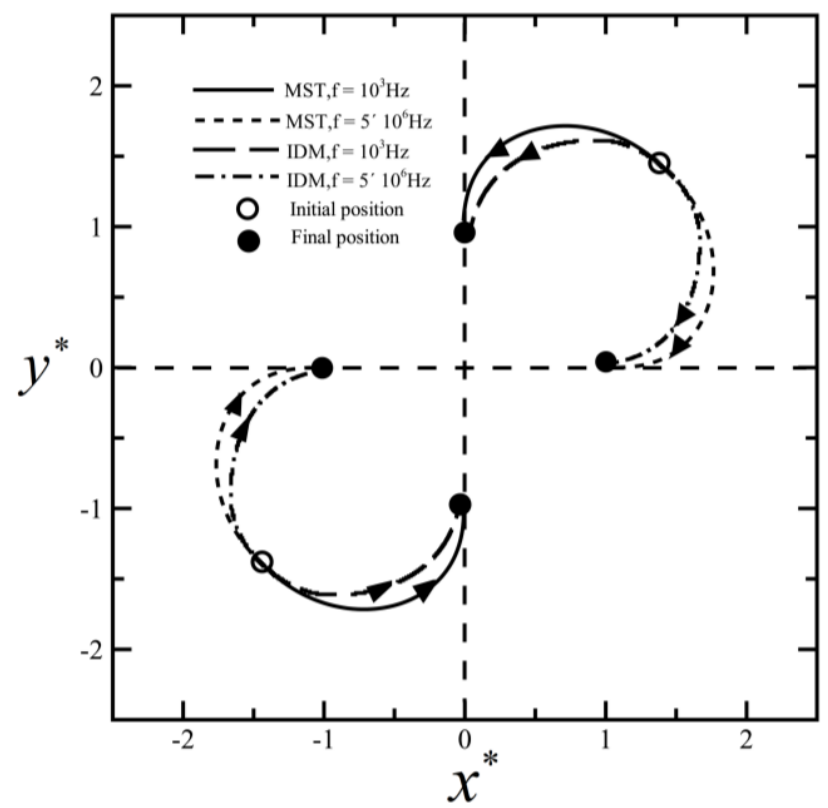

Fig.6 The comparison of the particles trajectories calculated by IDM method and MST method. Two particles are initially positioned at $\theta=45^{\circ}$ and $R^{*}=4$, and two different frequencies $f=10^{3} \mathrm{~Hz}$ and $f=5 \times 10^{6} \mathrm{~Hz}$ are respectively applied. Finally particle chains parallel or perpendicular to electric field are formed.

\section{Multi-particle interactions and particle chains in an electrical field}

DEP interactions of four, five and thirty particles will be investigated in this section, the effects of the frequency on the particle chains are investigated in particular.

\subsection{The DEP interaction of four particles in an uniform AC field}

Four particles $\left(a_{i}^{*}=1, i=1,4\right)$ are located in an uniform AC electric field of $\tilde{E}_{0}^{*}=0.05$. The initial particle locations are specified as $\left(r_{i}^{*}, \theta_{i}\right)$ in polar coordinate system, where $r_{i}^{*}=r_{i} / a$ is the dimensionless distance from the system origin to the $i$ th particle centers, $\theta_{i}$ is the angle of origin-center line of the $i$ th particle and $\mathrm{x}$ axis as shown in Fig.7a. The conductivity and the permittivity of the fluid are $\sigma_{f}=1.0 \times 10^{-3} \mathrm{~S} / \mathrm{m}, \varepsilon_{f}=78 \varepsilon_{0}$, respectively. The permittivities of the particles are $\varepsilon_{i}=2.5 \varepsilon_{0}(i=1,4)$ and the conductivities of the particles are $\sigma_{i}=3 \times 10^{-4} \mathrm{~S} / \mathrm{m}(i=1,4)$, respectively. The frequency of electric field is $f=10^{3} \mathrm{~Hz}$, the four particles are $\mathrm{N}$ particles, see Fig.3.

The particle trajectories and the final particle chains are shown in Fig.7 (b-d), where the dashed circles are the initial particle locations, the solid circles are the final particle chains and the crooked solid lines are the particle trajectories. It can be seen that when initial locations are $r_{i}^{*}=3,(i=1,4)$, $\theta_{1}=0^{\circ}, \theta_{2}=90^{\circ}, \theta_{3}=180^{\circ}, \theta_{4}=270^{\circ}$, the particles 2 and 4 move in the y direction, while the particle 1 
and 3 move in the $x$ direction due to the symmetry of initial locations. Finally the four particles are symmetrically clustered, as shown in Fig.7b, which is an unstable particle group. When a very small disturbance is applied to the initial position of particle $2\left(\theta_{2}=90^{\circ} \pm \delta, \delta=0.01^{\circ}\right)$, the particles experience asymmetric DEP forces, move in asymmetric trajectories, and finally form a particle chain aligned with the electrical field, as shown in Fig.7c, 7d.
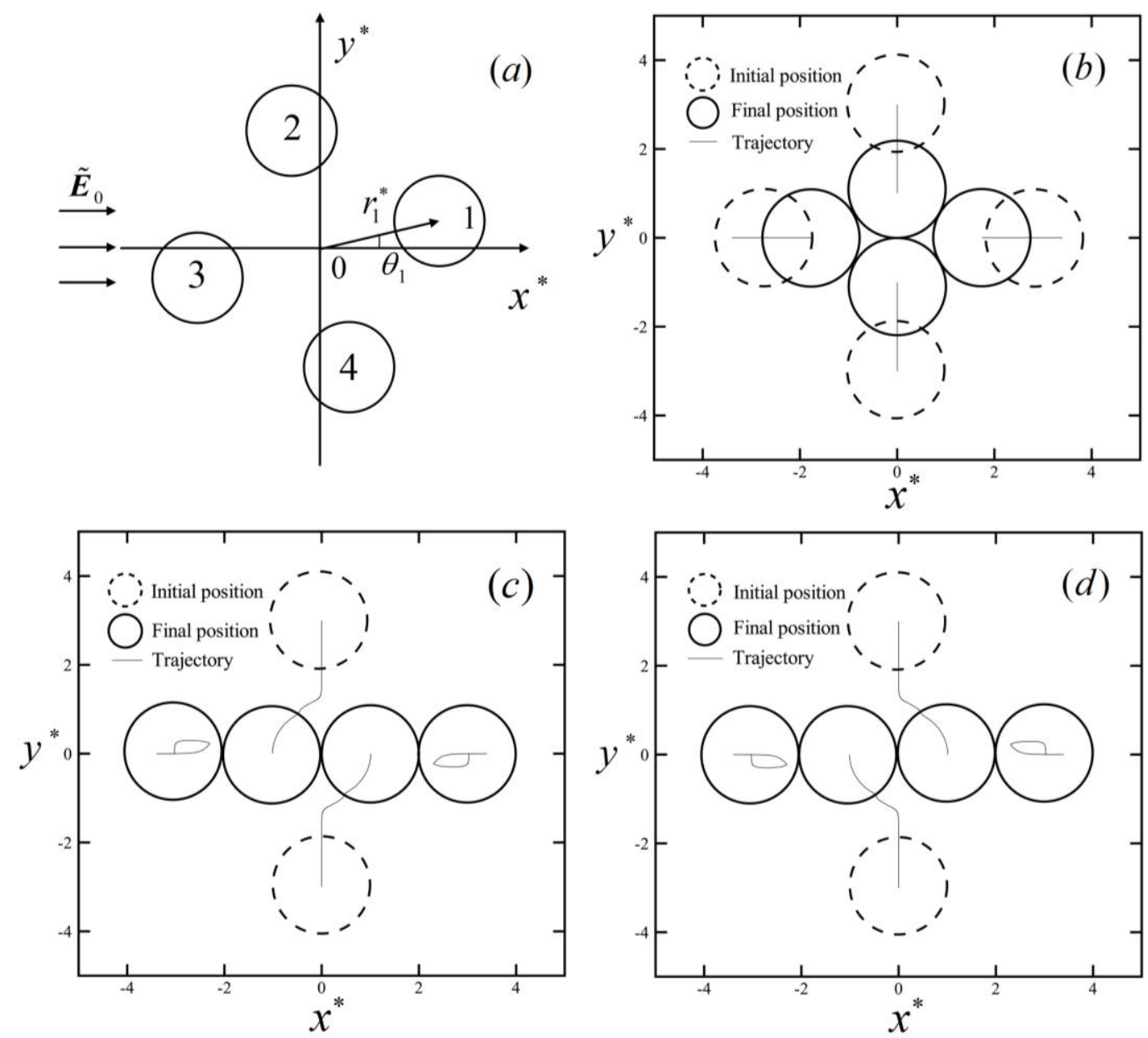

Fig.7 The initial configuration of four particles with $\varepsilon_{i}=2.5 \varepsilon_{0}(i=1,4)$ and $\sigma_{i}=3 \times 10^{-4} \mathrm{~S} / \mathrm{m}(i=1,4)$ in a uniform AC electric field with $f=10^{3} \mathrm{~Hz}(\mathrm{a})$ and the distinct trajectories and final particle chains under different status: (b) no disturbance. (c) $\theta_{2}=90^{\circ}+\delta, \delta=0.01^{\circ}$, counterclockwise disturbance. (d) $\theta_{2}=90^{\circ}-\delta$, clockwise disturbance.

\subsection{The DEP interaction of five particles}

The fifth particle is located at the origin of Fig.7a. The initial locations of five particles are $: r_{1}^{*}=r_{3}^{*}=2.5, r_{2}^{*}=r_{4}^{*}=4.5, \theta_{1}=45^{\circ}, \theta_{2}=135^{\circ}, \theta_{3}=225^{\circ}, \theta_{4}=315^{\circ}$. The conductivities of the particles $\sigma_{1}=\sigma_{3}=5 \times 10^{-3} \mathrm{~S} / \mathrm{m}, \sigma_{2}=\sigma_{4}=\sigma_{5}=3 \times 10^{-4} \mathrm{~S} / \mathrm{m}$, while the particle permittivities are the same as the previous section $3.1\left(\varepsilon_{i}=2.5 \varepsilon_{0}, i=1,5\right)$. Two particles are positive particles and three particles are negative particles when $f=10^{3} \mathrm{~Hz}$ (Fig.8a). All the particles are negative particles when $f=10^{7} \mathrm{~Hz}$ (Fig.8b). The initial positions, motion trajectories and final particle chains are shown in Fig. 8 (a-b), where the white circles are negative particles and the black circles are positive particles. It can be seen that the five dissimilar particles form a particle chain perpendicular to the electric field 
when $f=10^{3} \mathrm{~Hz}$, as shown in Fig.8a, and a particle chain parallel to the electric field is formed when $f=10^{7} \mathrm{~Hz}$ as shown in Fig. 8 b.
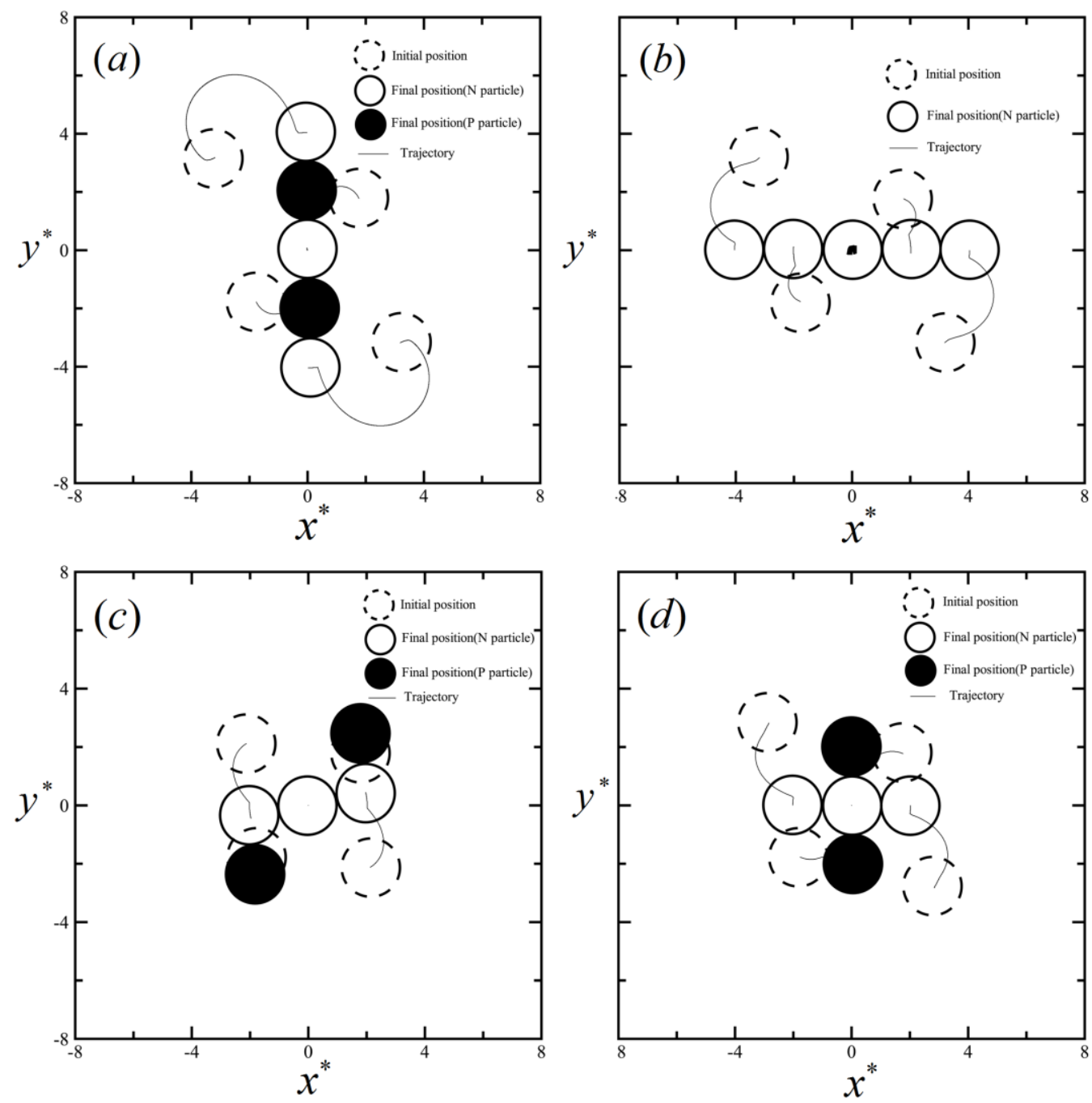

Fig.8 The initial locations and final particle chains of five particles interaction under different frequencies: (a) $f=10^{3} \mathrm{~Hz}$,

(b) $f=10^{7} \mathrm{~Hz}$, and the different patterns of particle chains caused by the changes of the initial positions and particle properties respectively at $f=10^{3} \mathrm{~Hz}$ compared to (a) are shown in (c) The particle chain by changes of initial positions, $r_{1}^{*}=r_{3}^{*}=2.5, r_{2}^{*}=r_{4}^{*}=3, r_{5}^{*}=0$ (compared to $\left.r_{1}^{*}=r_{3}^{*}=2.5, r_{2}^{*}=r_{4}^{*}=4.5, r_{5}^{*}=0\right)$, (d)The particle chain by the changes of particle conductivities, $\sigma_{1}=\sigma_{2}=3 \times 10^{-4} \mathrm{~S} / \mathrm{m}, \sigma_{2}=\sigma_{4}=\sigma_{5}=5 \times 10^{-3} \mathrm{~S} / \mathrm{m}$ (compared to $\left.\sigma_{1}=\sigma_{3}=5 \times 10^{-3} \mathrm{~S} / \mathrm{m}, \sigma_{2}=\sigma_{4}=\sigma_{5}=3 \times 10^{-4} \mathrm{~S} / \mathrm{m}\right)$.

Previous studies [13-17] have shown that two N-P (one is N and another is P) particles will form a particle chain perpendicular to the electric field, and two N-N(or P-P) particles will form a particle chain parallel to the electric field. However, multiple particles (N-N, P-P or N-P) are not always forming a particle chain perpendicular to or parallel to the electric field. The patterns of particle chain can be different depending on their initial positions before the electrical field is applied, or the physicochemical properties of particles and fluids.

Keep the same parameters as the Fig.8a (two $\mathrm{P}$ and three $\mathrm{N}$ particles), except the locations of particle 2, 4 are slightly changed to $r_{2}^{*}=r_{4}^{*}=3$, the final particle chain at $f=10^{3} \mathrm{~Hz}$ is shown in Fig. $8 \mathrm{c}$, which 
is totally different from the Fig.8a. It can be seen that the similar particles 2, 4, 5 form a chain nearly parallel to the electric field, while the dissimilar particles 1, 4 and the particles 2, 3 form chains perpendicular to the electric field.

Keep the same parameters as the Fig.8a and the same initial locations, but the conductivities of the particles are changed to $\sigma_{1}=\sigma_{3}=3 \times 10^{-4} \mathrm{~S} / \mathrm{m}, \sigma_{2}=\sigma_{4}=\sigma_{5}=5 \times 10^{-3} \mathrm{~S} / \mathrm{m}$, the final particle chain at $f=10^{3} \mathrm{~Hz}$ are shown in Fig.8d. It can be seen that the similar particles 2, 4, 5 form a chain parallel to the electric field, while the dissimilar particles 1, 3, 5 form a chain perpendicular to the electric field. Many different particle chains can be formed in multiple particles interactions depending on different physicochemical properties of particles and fluids and initial locations of the particles, but the essential behaviors of particle chain (or cluster) remain the same, as observed in experiments. It will not be presented in the current work.

Furthermore, the chains/clusters of multiple particles are not always perfectly parallel or perpendicular to the electric field due to the influence of the surrounding particles, as shown in Fig.8c, where the three N particles form a particle chain slightly sloped with the electric field. This phenomenon has also been observed in the experiments [31] for a case of a large number of randomly distributed particles in an electrical field.

\subsection{The DEP interaction of thirty particles}

Thirty particles are randomly suspended in the fluid. The particles consist of two types of particles with different permittivities and conductivities: (1) Fifteen particles of $\varepsilon_{1}=2.5 \varepsilon_{0}, \sigma_{1}=3 \times 10^{-4} \mathrm{~S} / \mathrm{m}$ (2) Fifteen particles of $\varepsilon_{2}=2.5 \varepsilon_{0}, \sigma_{2}=5 \times 10^{-3} \mathrm{~S} / \mathrm{m}$. The properties of the fluid are the same as of the previous section, dimensionless electric field is $\tilde{E}_{0}^{*}=0.05$. The initial positions are shown in Fig.9a, where the white circles denote the particles of $\sigma_{1}$, and the black circles denote particles of $\sigma_{2}$.

The particle chains at the frequencies of $f=\left(10^{3}, 10^{6}, 10^{7}\right) \mathrm{Hz}$ are shown in Fig.9 (b-d), where the white circles denote the $\mathrm{N}$ particles, the black circles denote P particles. $K_{R 1}=-0.5385, K_{R 2}=0.6667$ at frequencies $f=10^{3} \mathrm{~Hz}$, and $K_{R 1}=-0.9068, K_{R 2}=0.0926$ at $f=10^{6} \mathrm{~Hz}$. The particles consist of fifteen $\mathrm{N}$ particles and fifteen $\mathrm{P}$ particles at $f=\left(10^{3}, 10^{6}\right) \mathrm{Hz}$. The particles with larger $\left|K_{R}\right|$ experience stronger interaction forces and form longer chains than those with smaller $\left|K_{R}\right|$. At $f=10^{3} \mathrm{~Hz}$, the particles with $\left|K_{R 2}\right|=0.6667$ (black circles) form longer P-P particle chains parallel to the electric field than the N-N particle chains, as shown in Fig.9b. At $f=10^{6} \mathrm{~Hz}$, the particles with $\left|K_{R 1}\right|=0.9068$ (white circles) form longer N-N particle chains than the P-P particle chains, as shown in Fig.9c. The N particles and $\mathrm{P}$ particles attract each other, forming N-P particle chains perpendicular to the electric field, as shown in Fig.9b. At $f=10^{7} \mathrm{~Hz}, K_{R 1}=-0.9376, K_{R 2}=-0.9096$, the particles are all $\mathrm{N}$ particles, finally form three longer N-N particle chains parallel to the electric field, as shown in Fig.9d. The particle chains experience repelling forces perpendicular to the electrical field. 


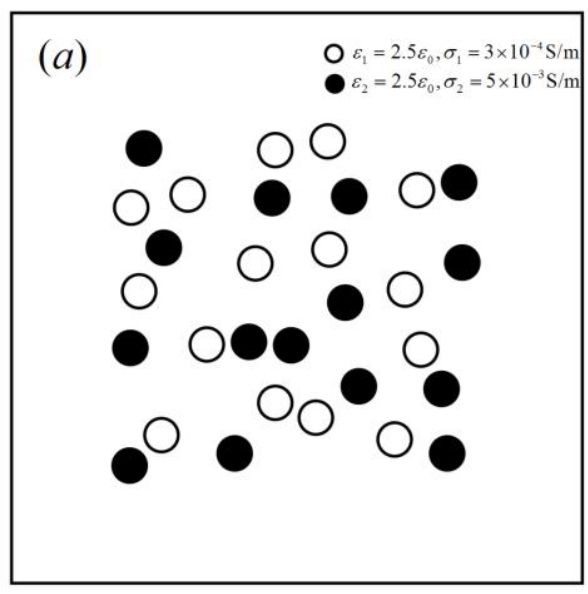

$(b)$

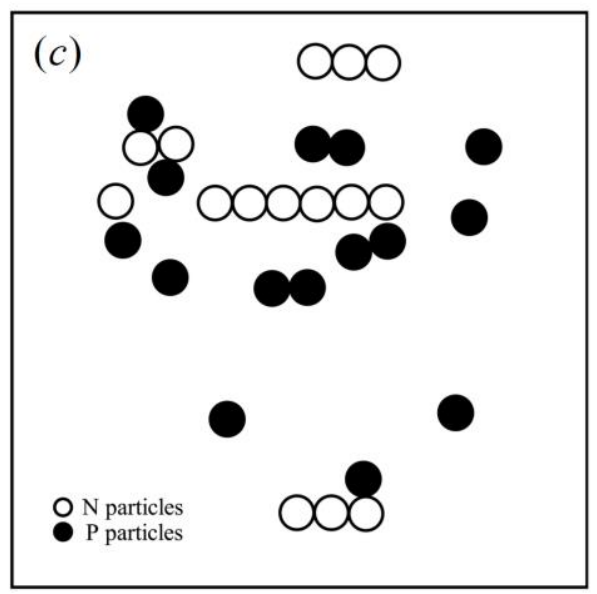

$(d)$

0000000000

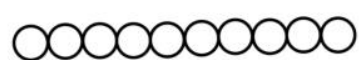

\section{0}

$\mathrm{ON}$ particles

Fig.9 The initial random distribution (a) and final particle chains at different frequencies (b-d) of thirty particles in a uniform $\mathrm{AC}$ electric field. The white circles and black circles in (a) denote $\varepsilon_{1}=2.5 \varepsilon_{0}, \sigma_{1}=3 \times 10^{-4} \mathrm{~S} / \mathrm{m}$ and

$\varepsilon_{2}=2.5 \varepsilon_{0}, \sigma_{2}=5 \times 10^{-3} \mathrm{~S} / \mathrm{m}$, respectively, while those in (b-d) denote $\mathrm{N}$ particles and $\mathrm{P}$ particles, respectively. The frequencies of (b-d) are $f=10^{3} \mathrm{~Hz}, f=10^{6} \mathrm{~Hz}, f=10^{7} \mathrm{~Hz}$, respectively.

Above simulated particle chains are compared with the experimental results of Giner's work in 1999 [31], as shown in Fig.10, where mixture of yeast cells and polystyrene microspheres are placed in a uniform AC electric field. It can be seen that the essential behaviors of simulated particle chains in this work are well consistent with experimental observation where the particle chains of polystyrene microspheres (white circle) are more significant at high frequency $f=10^{5} \mathrm{~Hz}$ than those at low frequency $f=50 \mathrm{~Hz}$. 
(a)

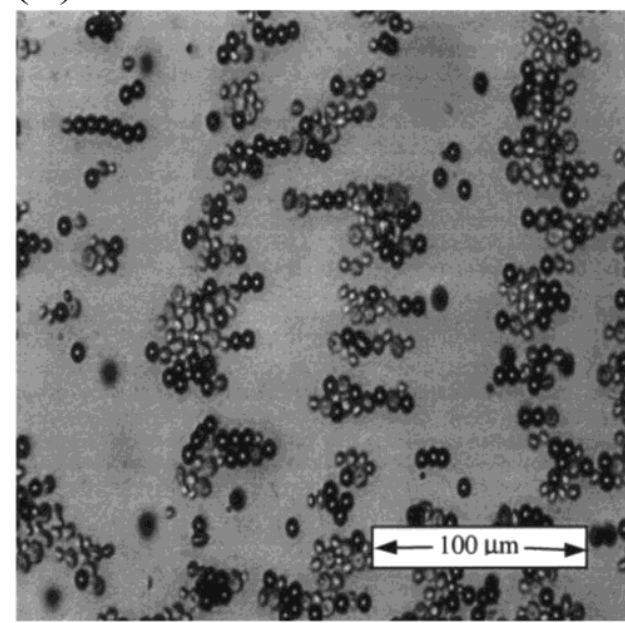

(b)

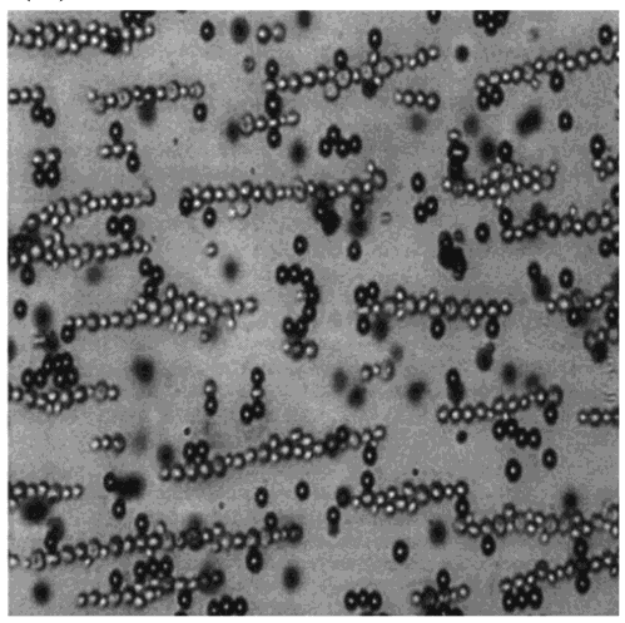

Fig.10 The experimental results of particle interaction in a uniform AC electric field.

The particles are mixture of yeast cells and polystyrene beads which have different C-M factors. (a) $f=50 \mathrm{~Hz}$, (b) $f=10^{5} \mathrm{~Hz}$, quoted from Giner et al (1999).

\section{Conclusions}

An iterative dipole moment (IDM) method to study multiple particles interactions in AC electric field is proposed in this work. The IDM method is an analytic algorithm without complicated numerical computations for solving differential equations of electrical field, and simple to implement. The DEP forces of particle interactions in a uniform AC electrical field calculated by IDM are generally in good agreement with MST method. The simulated particle chains (or clusters) by IDM method indicate that the particle trajectories and patterns of particle chains can be various styles depending on the initial particle locations and the physical properties of the particles. Arbitrary small disturbance can result in totally different particle chains (clusters).

A larger number of randomly distributed particles in an AC field can form different patterns of particle chains at different frequencies. By properly tuning the frequency of the electric field, the positive and negative particles can be mutually transformed resulting to different particle chains (clusters). In particular, the particles could be all positive or all negative in a specific region of frequency, finally form several long particle chains parallel to the electrical field. A frequency tuning of AC field can be an effective way to manipulate particles in a variety of applications.

\section{References}

[1] Caruso F. Colloids and colloid assemblies: synthesis, modification, organization and utilization of colloid particles. 2004.

[2] Markx G H, Carney L, Littlefair M, et al. Recreating the hematon: microfabrication of artificial haematopoietic stem cell microniches in vitro using dielectrophoresis. Biomedical microdevices, 2009, 11(1): 143-150.

[3] Velev O D, Kaler E W. In situ assembly of colloidal particles into miniaturized biosensors. Langmuir, 1999, 15(11): 3693-3698. 
[4] Pohl, H. A. Dielectrophoresis. Cambridge University Press, Cambridge, England, 1978.

[5] Al-Jarro A, Paul J, Thomas D W P, et al. Direct calculation of Maxwell stress tensor for accurate trajectory prediction during DEP for 2D and 3D structures. Journal of Physics D: Applied Physics, 2007, 40(1): 71-77.

[6] Rosales C, Lim K M. Numerical comparison between Maxwell stress method and equivalent multipole approach for calculation of the dielectrophoretic force in single - cell traps. Electrophoresis, 2005, 26(11): 2057-2065.

[7] Wang X, Wang X B, Gascoyne P R C. General expressions for dielectrophoretic force and electrorotational torque derived using the Maxwell stress tensor method. Journal of electrostatics, 1997, 39(4): 277-295.

[8] Kang K H, Li D. Dielectric force and relative motion between two spherical particles in electrophoresis. Langmuir, 2006, 22(4): 1602-1608.

[9] House D L, Luo H, Chang S. Numerical study on dielectrophoretic chaining of two ellipsoidal particles. Journal of colloid and interface science, 2012, 374(1): 141-149.

[10] Ai Y, Joo S W, Jiang Y, et al. Transient electrophoretic motion of a charged particle through a converging-diverging microchannel: Effect of direct current-dielectrophoretic force. Electrophoresis, 2009, 30(14): 2499-2506.

[11] Ai Y, Beskok A, Gauthier D T, et al. DC electrokinetic transport of cylindrical cells in straight microchannels. Biomicrofluidics, 2009, 3(4): 044110.

[12] Ai Y, Park S, Zhu J, et al. DC electrokinetic particle transport in an L-shaped microchannel. Langmuir, 2009, 26(4): 2937-2944.

[13] Ai Y, Qian S. DC dielectrophoretic particle-particle interactions and their relative motions. Journal of colloid and interface science, 2010, 346(2): 448-454

[14] Kang S, Maniyeri R. Dielectrophoretic motions of multiple particles and their analogy with the magnetophoretic counterparts. Journal of mechanical science and technology, 2012, 26(11): 3503-3513.

[15] Hossan M R, Dillon R, Roy A K, et al. Modeling and simulation of dielectrophoretic particle-particle interactions and assembly. Journal of colloid and interface science, 2013, 394: 619-629.

[16] Kang S. Dielectrophoretic motion of two particles with diverse sets of the electric conductivity under a uniform electric field. Computers \& Fluids, 2014.

[17] Chuanchuan Xie, Bo Chen, Chiu-On Ng, Xinping Zhou, Jiankang Wu, Numerical study of interactive motion of dielectrophoretic particles, European Journal of Mechanics B/Fluids, 2015,49: 208-216.

[18] Kurgan E, Gas P. Calculation of Forces Imposed on Particles in AC Dielectrophoresis. Przegląd Elektrotechniczny, 2009, 85: 100-103.

[19] Hossan M R, Dillon R, Dutta P. Hybrid immersed interface-immersed boundary methods for AC dielectrophoresis. Journal of Computational Physics, 2014, 270: 640-659.

[20] Ai Y, Zeng Z, Qian S. Direct numerical simulation of AC dielectrophoretic particle-particle interactive motions. Journal of colloid and interface science, 2014, 417: 72-79.

[21]T. B. Jones, R. D. Miller, K. S. Robinson, W. Y. Fowlkes, Multipolar interactions of dielectric spheres, Journal of Electrostatics, 1989, 22(3): 231-244. 
[22] Masao Washizu, T. B. Jones, Multipolar dielectrophoretic force calculation, Journal of Electrostatics, 1994, 33(2): 187-198.

[23] Masao Washizu, T. B. Jones, Generalized multipolar dielectrophoretic force and electrorotational torque calculation, Journal of Electrostatics, 1996, 38(3): 199-211.

[24] T. B. Jones, Masao Washizu, Multipolar dielectrophoretic and electrorotation theory, Journal of Electrostatics, 1996, 37(1-2): 121-134.

[25] Yoji Nakajima, Tatsushi Matsuyama, Electrostatic field and force calculation for a chain of identical dielectric spheres aligned parallel to uniformly applied electric field, Journal of Electrostatics, 2002, 55(2): 203-221.

[26]H. Moncada-Hernandez, M. Nagler and A. Minerick, Theoretical and experimental examination of particle-particle interaction effects on induced dipole moments and dielectrophoretic responses of multiple particle chains, Electrophoresis, 2014,35,1803-1813

[27] Liu L, Xie C, Chen B, et al. Iterative dipole moment method for calculating dielectrophoretic forces of particle-particle electric field interactions. Applied Mathematics and Mechanics, 2015, 36(11): 1499-1512.

[28] Liu L, Xie C, Chen B, et al. A new method for the interaction between multiple DEP particles: iterative dipole moment method. Microsystem Technologies, 2015: 1-10.

[29] Liu L, Xie C, Chen B, et al. Numerical study of particle chains of a large number of randomly distributed DEP particles using iterative dipole moment method. Journal of Chemical Technology and Biotechnology, 2015.

[30] Hywel Morgan and Nicolas G Green, AC Electrokinetics: colloids and nanoparticles, Research Studies Press Ltd. England,2003, Page 62.

[31] V. Giner, M. Sancho, R. S. Lee, G. Martinez and R. Pethig, Transverse dipolar chaining in binary suspensions induced by rf fields, J. Phys. D: Appl. Phys. 1999, 32: 1182-1186.

[32] KURGAN E. Calculation of Forces in AC Dielectrophoretic Separation. Przegląd Elektrotechniczny, 2013, 89. 